\title{
À quoi sert la dot ? Aliénations dotales, économie familiale et stratégies des couples à Turin au XVIII ${ }^{\mathrm{e}}$ siècle
}

\section{Par Beatrice Zucca Micheletto}

Une présence constante dans la vie des couples

D'un point de vue historiographique, la dot et sa fonction ont été des sujets longuement traités par l'anthropologie et le droit avant de devenir, par la suite, un terrain de recherche de l'histoire sociale et notamment un des piliers de l'histoire de la famille ${ }^{1}$.

En s'intéressant à la dot dans le cadre de l'analyse des logiques de transmissions patrimoniales, un premier courant de recherches a mis en relief son rôle symbolique et relationnel ainsi que sa fonction de marqueur du statut social. Selon cette perspective, la dot est donc un patrimoine utile pour réaliser les politiques d'alliance des familles et satisfaire leurs ambitions de mobilité sociale. D'après ces études, les biens dotaux circulent entre les familles et tissent des liens de parenté et de collaboration qui sont à la fois symboliques et matériels. Il s'agit surtout d'une perspective de recherche qui privilégie les couches sociales aisées ou les familles clairement insérées dans un parcours de mobilité sociale ${ }^{2}$. En effet, la valeur de la dot qu'une famille est en mesure d'établir, conditionne les opportunités matrimoniales de la fille et donc le choix du milieu social dans lequel le mariage aura lieu. Si la dot est élevée, parfois supérieure au train de vie réel de la famille, cela traduit une volonté de mobilité sociale, et l'espoir de réaliser une alliance avec une famille d'un niveau social égal ou supérieur. En sens inverse, un époux issu d'une famille appauvrie ou déclassée, ou non intégrée dans les réseaux sociaux de la communauté, doit probablement se contenter d'un mariage « vers le bas » de l'échelle sociale et donc, vraisemblablement, d'une dot modeste ${ }^{3}$.

\footnotetext{
1 Je tiens à remercier les évaluateurs anonymes de la revue pour leur lecture et leurs remarques.

2 L'historiographie consacrée à la dot, aux systèmes normatifs et aux enjeux d'ordre social, symbolique et économique, est extrêmement vaste. Les études repères sur le sujet restent (Goody, 1973), (Owen Hughes, 1978), (Klapisch-Zuber, 1990) et (Siegrist, Samudio, 2006).

3 Des nombreuses études - consacrées principalement à Venise et à Florence - ont bien éclairci ces dynamiques sociales (Klapisch-Zuber, 1990 ; Chojnacki, 2000 ; Bellavitis, 2001).
} 
Au fil des années, bien d'autres études ont abordé le rôle de la dot soit au moment de sa constitution, soit au moment de la mort du mari, lorsque la veuve peut réclamer ses droits et la récupérer.

Plusieurs travaux se sont concentrés sur les protagonistes (institutions ou individus) chargés de constituer une dot et de la fournir aux filles. Dans les sociétés de l'Ancien Régime, la dot était indispensable pour avoir accès au marché matrimonial, dans toutes les couches sociales; dans ce but, de nombreuses institutions publiques ou privées, laïques ou religieuses, prenaient en charge le paiement d'une dot. A Rome, par exemple, jusqu'au XIX ${ }^{\mathrm{e}}$ siècle, un système ramifié d'institutions religieuses se donnait pour objectif de constituer les dots pour les filles orphelines ou abandonnées qui y étaient hébergées, ainsi que pour des filles vivant à l'extérieur qui présentaient leur candidature (Groppi, 1994 ; D’Amelia, 1990). Il en était de même à Bologne, où les « Opere pie dotali », des institutions directement gérées par le Mont de Piété de la ville, étaient chargées d'aider les jeunes filles à se constituer une dot, lorsque les seules ressources familiales n'étaient pas suffisantes (Chabot, Fornasari, 1997). De la même manière, dans le Royaume de Naples entre le $\mathrm{XVI}^{\mathrm{e}}$ et le $\mathrm{XVIII}{ }^{\mathrm{e}}$ siècle, les «monti di maritaggio » pouvaient être soit privés, constitués à travers un legs testamentaire et destinés à doter les filles d'un même lignage, soit publics et donc ouverts à toutes les filles démunies de la communauté (Delille, 1982).

Mais la dot attire aussi l'attention des chercheurs à l'autre extrémité du cycle de vie du couple. Le prédécès du mari et l'entrée de la femme dans sa condition de veuve soulèvent la question de la restitution de la dot qui devait être effectuée par les héritiers de l'époux. Comme plusieurs études le montrent, l'état de veuve mettait la femme dans une position ambiguë. D’une part, elle pouvait jouir des nouvelles libertés juridiques que le statut de femme mariée lui interdisait, d'autre part, elle risquait l'isolement relationnel et économique et donc un passage rapide vers une situation de pauvreté. La possibilité de récupérer les biens dotaux et de les conserver ou éventuellement de se remarier, était un enjeu fondamental qui déterminait la destinée de la veuve. D'ailleurs, le moment et les modalités de la restitution de ces biens n'étaient pas un passage anodin dans la vie familiale et la veuve était parfois obligée d'engager une action en justice pour réussir à les récupérer ${ }^{4}$.

$4 \quad$ La bibliographie est très vaste. Voir notamment (Diefendorf, 1982), (Klapisch-Zuber, 1983), (Cavallo, Warner, 1999), (Juratic, 1999), (Chojnacki, 2000), (Beauvalet-Boutouyrie, 2001), (FauveChamoux, 2002), (Pellegrin, Winn, 2003), (Bellavitis, 2008). 
$\mathrm{Si}$ toutes les études et les recherches évoquées soulèvent des problèmes historiographiques très importants, elles n'abordent pourtant pas la question de l'emploi des biens dotaux pendant le cycle de vie du couple. Que se passait-il après le paiement d'une dot ? Et par la suite, comment la dot était-elle utilisée au sein du ménage ? A quoi servait la dot pendant la vie conjugale ? Aucune étude n'a jamais retracé cette histoire. On sait pourtant que pendant la vie conjugale, les biens dotaux étaient normalement investis ; le couple percevait des intérêts légaux qui, officiellement, devaient servir à couvrir les charges et les frais de la vie matrimoniale.

Il est vrai aussi que certaines études ont mis en lumière le rôle économique de la dot pendant la vie conjugale. Ce patrimoine était un enjeu pour la survie de la nouvelle famille ${ }^{5}$. En Italie, dès le $\mathrm{XVI}^{\mathrm{e}}$ siècle et jusqu'à une longue partie du $\mathrm{XIX}^{\mathrm{e}}$ siècle, parmi les couches artisanales et commerçantes, la dot avait pour objectif de soutenir l'activité des jeunes mariés et même d'en enclencher le démarrage, ou encore de permettre à l'activité familiale de se rétablir après une période de difficultés. Toutefois, au delà de quelques exceptions, la plus grande partie des recherches que nous avons évoquées ne développe pas une véritable analyse du sujet et de nombreuses questions historiographiques demeurent toujours sans réponse.

C'est donc à partir d'une telle perspective que cet article envisage de réfléchir explicitement à l'usage économique de la dot pendant la vie conjugale. Il s'agit de montrer comment, quand et dans quelles phases du cycle de vie des couples, ce capital était employé. Enfin, nous chercherons à esquisser des modèles de comportement et à expliquer concrètement les contextes dans lesquels cette utilisation avait lieu.

L'image d'une dot conçue comme un patrimoine «immobile» et destiné à être thésaurisé, peut être aisément remise en cause lorsque l'on s'intéresse à l'utilisation des biens dotaux qui avait lieu par le biais d'une procédure d'aliénation dotale. En théorie, les biens placés sous le régime dotal étaient inaliénables, c'est-à-dire qu'ils ne pouvaient être ni vendus, ni laissés, ni encore remis à titre gratuit à quiconque. Toutefois, selon le droit romain, la dot était constituée pour permettre aux conjoints de soutenir les dépenses et les charges du mariage. Ce principe avait été accueilli formellement par la

\footnotetext{
5 Giovanni Levi a expliqué que dans le monde rural piémontais, la dot (constituée par un ou plusieurs biens fonciers) et non pas l'héritage, marquait le commencement de l'économie domestique des nouveaux couples (Levi, 1976) ; il en est de même pour les Pouilles entre le XVI ${ }^{\mathrm{e}}$ et le XVIII ${ }^{\mathrm{e}}$ siècle (Delille, 1985). Sur la fonction économique de la dot, voir aussi (Owen Hughes, 1979), (Fazio, 1992), (Scardozzi, 1998) et (Lanaro, Varanini, 2009).
} 
Charte constitutionnelle de l'Etat piémontais, à tel point qu'à travers une procédure juridique, les couples pouvaient obtenir une dérogation sur l'inaliénabilité des biens dotaux (ou sur une partie d'entre eux), et donc les vendre et les transformer en argent liquide prêt à être dépensé. Les actes d'aliénation dotale montrent en conséquence l'usage économique de la dot et son rôle de ressource.

Bien entendu, cette perspective d'analyse ne veut pas dénier à la dot sa valeur symbolique et relationnelle; comme l'écrit Ida Fazio (1992, 293), « une dot reste nécessaire pour conférer un support économique et une dignité symbolique à l'union conjugale $»^{6}$. Toutefois - comme les actes d'aliénation dotale le démontrent - l'image d'un trésor jalousement conservé et destiné à circuler, inaltéré, à travers les générations ne correspond pas non plus à la réalité des dots dont on connaît le destin.

Afin d'approfondir cette conviction, j'ai étudié un corpus de 146 procès d'aliénation des biens dotaux, passés à Turin entre 1765 et 1780. Il s'agit d'un type de documentation jusqu'à maintenant négligé par les historiens et sur lequel les études demeurent rares. Dans les archives de Gio' Batta Franco - un des notaires qui occupaient des fonctions de secrétaire au sein du tribunal civil de la Giudicatura -, on compte entre 1740 et 178519 actes par an en moyenne, avec des pics de plus de 30 procès pour les années 1768, 1769, 1773, 1775 et 1780 (ce qui justifie aussi mon choix de constituer un échantillon de procès dans la période 1765-1780). On peut estimer que l'effectif de ce type de procédures pourrait être multiplié par trois si l'on considérait les autres tribunaux civils - notamment la Préfecture et le Sénat - chargés eux aussi de s'occuper d'aliénations dotales.

J'analyserai dans un premier temps ce corpus d'actes d'aliénation. Par le biais de la procédure juridique et des motivations de la demande, je mettrai en lumière une caractéristique essentielle des biens dotaux, celle d'être non seulement un patrimoine destiné aux « jeux d'échanges » des femmes mais encore - voire surtout - une ressource économique multidimensionnelle et polyfonctionnelle du ménage.

Par ailleurs, l'analyse de ces sources permettra de réfléchir d'une façon plus ample à l'usage des institutions d'Ancien Régime de la part des individus. En effet, les demandes d'aliénations dotales nous font réfléchir sur la volonté des acteurs - et notamment sur le rôle de la femme mariée - d'aliéner ces biens, sur leurs marges de choix et sur les contraintes juridiques d'une telle démarche.

$6 \quad$ C'est moi qui traduis. 
Dans les sociétés d'Ancien Régime, la condition maritale marquait la position sociale, juridique et économique des femmes, ainsi que les espaces et les opportunités qui leur étaient offerts ${ }^{7}$. Or, ces actes d'aliénation dotale fournissent l'occasion de s'interroger sur l'existence d'éventuelles stratégies d'utilisation de la dot venant de la part de la femme mariée ou des conjoints ensemble. Finalement, ils montrent la capacité des épouses à prendre des initiatives et peut-être à faire des choix ; c'est-à-dire, en somme, d'être des sujets actifs par rapport à leur patrimoine et à la gestion de l'économie familiale, plus actifs que ce que certains lieux communs de l'historiographie laissent à penser.

\section{La dot en Piémont}

À l'instar d'autres pays d'Europe occidentale, le régime dotal piémontais était le résultat d'un mélange et d'une coexistence entre la loi étatique, le droit romain, la coutume et les statuts de chaque ville ${ }^{8}$. La dot était apportée par la femme (et sa famille) lors du mariage de celle-ci.

Au contraire d'autres réalités urbaines et rurales (Levi, 1976; Delille, 1985 ; Bellavitis, 2001), à Turin pendant le XVIII ${ }^{\text {ème }}$ siècle, la dot était payée principalement en argent ou avec des écritures privées, ou encore avec des coupons de rente de la dette publique (les « luoghi di Monte »), et seulement d'une façon sporadique avec des biens immeubles - maisons ou terres. De plus, parmi les couches sociales populaires, on trouve assez fréquemment des dots entièrement - ou presque - payées en biens meubles. Parfois, il s'agissait des biens du trousseau de mariage : vêtements, linges de corps et bijoux destinés à l'épouse ; mais souvent on y recense aussi des meubles, de la vaisselle, des ustensiles, du linge de maison, des outils destinés à équiper la maison des époux et à leur permettre de démarrer ou de continuer à gérer une activité commerciale ou artisanale. À titre indicatif, dans un échantillon de 174 actes de dotation passés à Turin entre 1760 et 1780 dans toutes les couches sociales, on ne compte que trois dots

$7 \quad$ Sur le travail et/ou la propriété des femmes mariées, voir (Fazio, 1992; 1996), (Ago, 1996; 1998), (Groppi, 1996), (Arru, 1998a), (Calvi, Chabot, 1998), (Howell, 1998), (Abreu-Ferreira, 2000), (Palazzi, 2002), (Ogilvie, 2003), (Feci, 2004), (Erickson, 2008), (Van Den Heuvel, Van Nederveen Meerkerk, 2008) et (Zucca Micheletto, 2008).

8 On trouvera des indications spécifiques sur le système dotal piémontais dans (Pene Vidari, 1980$81 ; 1983)$. 
constituées avec des immeubles et une avec des lopins de terre ${ }^{9}$; 13 d'entre elles ne comptaient que des biens meubles et les restantes furent payées soit avec de l'argent, soit avec la remise des coupons de « monti », soit avec une écriture privée.

Les biens dotaux (y compris le trousseau de mariage) étaient investis d'un statut juridique spécifique, car si le dominio - la nue propriété - revenait à la femme, la possessio - la gestion et la jouissance de son usufruit - était réservée au mari tout au long de la vie matrimoniale. Le mari (et parfois aussi son père) recevait donc formellement la dot; il pouvait en choisir la gestion et il devait surtout l'assurer sur ses biens. A son tour, ce capital pouvait être investi auprès d'un tiers qui s'engageait à payer les intérêts légaux annuels («l'implicatario ») ${ }^{10}$; autrement, il pouvait être investi dans les Monti di San Giovanni de la ville de Turin ${ }^{11}$. Parfois, si l'époux ne pouvait assurer la dot, elle restait confiée à la famille de l'épouse (chez le père, l'oncle ou le frère de celle-ci). Dans tous les cas, le couple devait pouvoir percevoir les intérêts légaux sur le capital dotal.

Les héritiers du mari étaient aussi tenus de garantir la restitution de la dot à la femme lorsqu'elle devenait veuve. C'est uniquement dans ce cas que la dot devenait une propriété de la veuve, qui pouvait alors la gérer librement.

Selon la coutume de la ville de Turin, au moment de la constitution de la dot par acte public devant le notaire, le mari ajoutait au capital initial une somme qui correspondait à un tiers ou un quart de sa valeur. Ces apports maritaux - qui ont leur origine dans l'ancien « don du matin » pratiqué chez les Lombards au Moyen-Âge étaient connus sous le nom d'« augmentum dotis » (augment de dot) et étaient ajoutés au capital dotal sur lequel le couple percevait les rentes; à la mort du mari, l'« augmentum dotis » passait également en propriété de la veuve (Klapisch-Zuber, 1990, 192 ; Bellomo, 1961).

Enfin, des dons de noces (venant du mari lui-même) complétaient l'ensemble des biens dotaux. Mais contrairement au capital dotal, ces biens étaient propriété de la

\footnotetext{
$9 \quad$ AST, Insinuazione di Torino.

10 L' « implicatario » - issu de la notabilité ou des arts libéraux et installé soit dans la ville de Turin, soit dans la communauté d'origine des époux - recevait formellement l'argent dotal et s'engageait à l'investir dans des activités pour en percevoir une rente, dont une partie était payée aux époux à titre d'intérêts légaux du capital dotal.

11 Les Monti di San Giovanni de la ville de Turin étaient des titres de la dette publique dont la gestion revenait à l'administration de la ville. Particuliers et institutions achetaient les titres («luoghi di monti ») et percevaient des intérêts légaux.
} 
femme qui, selon les dispositions maritales, pouvait les gérer librement d'ores et déjà pendant le mariage, et bien entendu après le décès du mari.

\section{Vendre les biens dotaux : ce que dit la loi piémontaise}

Les actes d'aliénation dotale étaient le résultat d'une procédure qui relevait de la justice civile et notamment de ce que les juristes du XIX ${ }^{\mathrm{e}}$ siècle définissaient comme de la « juridiction volontaire » (Dionisotti, 1881, 298).

La plus grande partie des procès qui permettaient aux individus de vendre les biens dotaux (ou une partie seulement) et de les transformer en argent liquide se passaient devant le tribunal civil de la ville de Turin, la Giudicatura. Ce tribunal prenait en charge les cas de justice civile concernant des individus à statut juridique spécifique et leur patrimoine : il s'occupait donc de la vente des biens dotaux, mais aussi de la vente des biens des mineurs et des religieux, des cas de clôture de tutelle des mineurs, des actes d'émancipation de jeunes de la patria potestas ${ }^{12}$.

Les décisions de cette procédure de justice devaient par la suite être enregistrés par acte public auprès des notaires agréés de la Giudicatura - notamment le secrétaire (segretario) et ses deux sous-secrétaires (sostituti segretari). C'est donc dans les archives des deux notaires qui travaillaient auprès du tribunal que j'ai repéré et établi un corpus de 146 actes d'aliénation dotale ${ }^{13}$.

Les biens dévolus à titre de dot jouissaient d'un statut patrimonial ambigu, à michemin entre biens à préserver et ressources susceptibles de soutenir les charges de l'économie domestique. Dans cet esprit, la loi piémontaise établissait que les biens dotaux pouvaient être libérés de leur inaliénabilité pour être vendus afin de financer une activité familiale ou de permettre aux conjoints de sortir d'une situation de difficulté économique. Les démarches nécessaires pour y parvenir comportaient plusieurs étapes et formaient un vrai parcours bureaucratique. Les dossiers que j'ai utilisés sont composés de plusieurs éléments : une supplique, une patente royale, la transcription de

\footnotetext{
12 La magistrature civile fut l'objet d'un renouvellement dans l'État piémontais à partir de l'action de Victor-Amédée II et dès la publication de la première Charte constitutionnelle de l'État en 1723. On pourra trouver toutes les réformes de la magistrature civile, ainsi que l'explication de ses tâches et fonctions dans (Duboin, 1818-1869) et (Dionisotti, 1881).

13 Il s'agit d'un échantillon issu d'un corpus plus large d'actes d'aliénation dotale, qui porte sur l'ensemble du XVIII siècle et sur lequel j'ai une recherche en cours. L'échantillon ici considéré porte sur les années 1765-1780. Archives Nationales de Turin (désormais AST), Notai di Torino, I versamento.
} 
l'interrogatoire de quatre témoins (« i testimoniali di informazione »), un décret du juge et un acte notarié.

Concrètement, la procédure commençait avec une supplique que les époux adressaient directement au roi, lequel devait à son tour se prononcer officiellement sur l'ouverture d'une procédure de justice auprès de la Giudicatura ${ }^{14}$.

Quelques semaines plus tard, le procès passait entre les mains de la magistrature civile qui était censée vérifier la réalité de l'état de nécessité du couple. Le juge interrogeait alors deux témoins (les « testimoni informanti ») qui pouvaient être liés par parenté à l'épouse (y compris la parenté par alliance) ou, à défaut, les voisins du domicile. Ensuite, le juge interrogeait deux témoins « comprovanti », soit deux hommes qui devaient à leur tour attester de la bonne réputation et de la fiabilité des deux premiers témoins. Finalement, si le juge se prononçait favorablement, un décret était passé et l'aliénation pouvait avoir lieu.

Après quelques semaines, c'était encore devant le juge et le secrétaire-notaire de la Giudicatura qu'avait lieu la dernière phase de l'aliénation. En fonction de la composition de la dot et de ses modes de placement, le couple vendait les « luoghi di Monte » ou recevait l'argent liquide de l'« implicatario » auquel il avait été confié ${ }^{15}$; ou bien encore, si une succession était en cours, le couple recevait l'argent directement des héritiers de la famille de l'épouse qui était censée payer la dot. Quoi qu'il en soit, à travers cet acte, le couple recevait la valeur des biens dotaux (tout ou partie) en argent liquide et, à son tour, délivrait une quittance de paiement.

En outre, dans le même acte, le couple était censé payer les dettes aux créanciers. En assurant un emploi immédiat de l'argent liquide, les institutions se protégeaient donc des éventuels abus ou gaspillages qui pouvaient dériver de la transformation de la dot en espèces. Après le paiement des dettes, s'il restait de l'argent

14 Selon la loi, la supplique au roi n'était pas nécessaire dans toutes les situations; le couple pouvait s'adresser directement à la Giudicatura. Pourtant, à ce stade de mon dépouillement, je n'ai repéré que des aliénations dotales qui débutaient par une supplique au roi. Il faut préciser aussi que la Giudicatura civile (la magistrature urbaine de la justice civile) n'était pas la seule institution chargée de délivrer le décret d'aliénation dotale. Selon la Charte constitutionnelle, les couples pouvaient s'adresser à d'autres magistratures, telles que la Préfecture ou le Sénat du Piémont, selon les motifs de la demande, le degré d'urgence de la situation, et la valeur de la dot. La Giudicatura était la magistrature civile la plus populaire à Turin sous l'Ancien Régime; c'est donc elle qui traitait une grande partie des demandes d'aliénation (Duboin, 1818-1869, Lib. III, Tit. III, 355-357). Sur l'importance des archives des magistratures urbaines civiles pour la recherche la plus récente, on se reportera à (Bellavitis, 2008) et (Cerutti, 2003).

$15 \quad$ Voir les notes 10 et 11. 
disponible, celui-ci était conservé par le couple qui s'engageait formellement à l'utiliser pour les dépenses de la famille.

Il convient d'ajouter que, selon la loi piémontaise, la vente de biens dotaux était équivalente à la vente de biens de mineurs et qu'elle devait se dérouler dans des conditions similaires. L'accord du mari-tuteur était donc indispensable ; à l'inverse, son absence était à l'origine du refus de la part des autorités de concéder l'aliénation.

Toutefois, puisque la propriété des biens dotaux revenait à la femme, l'aliénation ne pouvait avoir lieu qu'avec le consentement explicite de la femme. Au moment de la vente effective des biens, devant le notaire et le juge, la femme était interrogée à part et elle devait faire serment de n'avoir subi ni pression, ni obligation, ni « séduction $»{ }^{16}$.

Il est intéressant de remarquer que la vérification de l'accord et de la libre volonté de la femme ne fit l'objet d'une disposition législative que dans la première publication de la Charte constitutionnelle, en 1723 (Duboin, 1818-1869). Dans les chartes ultérieures (en 1729 et 1770), ce principe fut totalement abandonné, tandis que le parallélisme entre la vente des biens dotaux et celle des biens des mineurs demeura inchangé. Je reviendrai sur ce sujet par la suite; pour le moment, je me limiterai à observer que dans les actes conservés et enregistrés, il n'y a pas de cas d'opposition de la part de la femme.

\section{Les protagonistes}

Quelles sont les couches sociales qui avaient recours à l'aliénation dotale ?

Les tableaux 1 et 2 montrent que les métiers et les rangs sociaux concernés se répartissaient au sein d'un large spectre. Parmi les époux (tab. 1), les plus nombreux étaient les artisans et les commerçants (cordonniers, tailleurs, perruquiers, marchands de mode et de toiles, de riz, de blé et comestibles), suivis par les serviteurs et les domestiques de différents niveaux ; ensuite venaient les soldats et les officiers, et dans des proportions moindres, les employés (secrétaires et commis) et les professions libérales (avocats, notaires).

16 La formule employée était la suivante : « Presasi però prima in disparte [...] detta signora [nom et prénom de la femme] e da essa esplorato se sia stata indotta o sedotta nell'aver [...] umiliato detto ricorso a $\mathrm{Sm}$, la medema ha dichiarato e dichiara essere a ciò divenuta di suo libero e spontaneo volere», AST, Notai di Torino, I vers., vol. 2754, ff. 517r-523r. 
On peut établir un constat analogue du côté des épouses (tab. 2) : on y retrouve tout l'éventail des métiers typiques des femmes sous l'Ancien Régime : des couturières aux brodeuses, des serveuses aux petites marchandes de produits alimentaires.

\begin{tabular}{|c|c|}
\hline \multicolumn{2}{|l|}{ Tab 1. Métiers des hommes } \\
\hline serviteurs & 7 \\
\hline soldats & 7 \\
\hline tailleurs & 7 \\
\hline cordonniers & 5 \\
\hline perruquiers & 5 \\
\hline cafétiers / aubergistes & 4 \\
\hline fabricants d'eau de vie et confitures & 4 \\
\hline travailleurs à la campagna/jardiniers & 4 \\
\hline emploiés & 3 \\
\hline marchands de modes/toiles/merciers & 3 \\
\hline porte-faix & 3 \\
\hline quincailliers & 3 \\
\hline travailleurs de cuir et de peaux & 3 \\
\hline argentiers & 2 \\
\hline avocats & 2 \\
\hline bijoutiers & 2 \\
\hline cuisiniers & 2 \\
\hline dinandiers & 2 \\
\hline fabricants de bas & 2 \\
\hline fabricants de bonnets & 2 \\
\hline fabricants de pâtes & 2 \\
\hline marchands & 2 \\
\hline maréchal-ferrants & 2 \\
\hline notaires & 2 \\
\hline revendeurs de comestibles & 2 \\
\hline revendeurs de farines, graines et riz & 2 \\
\hline teinturiers & 2 \\
\hline armurier et soldat & 1 \\
\hline bouchers & 1 \\
\hline cardeurs & 1 \\
\hline fabricants de bâts & 1 \\
\hline fabricants de dentelles & 1 \\
\hline fabricants de matélas & 1 \\
\hline journaliers & 1 \\
\hline libraires & 1 \\
\hline marchands en papier & 1 \\
\hline ménusiers & 1 \\
\hline musiciens & 1 \\
\hline palefreniers & 1 \\
\hline peintres en papiers et toiles & 1 \\
\hline préposés aux écritures & 1 \\
\hline revedeurs de légumes et fruits & 1 \\
\hline
\end{tabular}




\begin{tabular}{|l|r|} 
saigneurs & 1 \\
\hline selliers & 1 \\
\hline serruriers & 1 \\
\hline tapissiers & 1 \\
\hline & \\
\hline sans travail & 1 \\
\hline métiers non connus & 40 \\
\hline tot & 146 \\
\hline
\end{tabular}

\begin{tabular}{|l|r|}
\hline Tab 2. Métiers des femmes \\
\hline couturières / brodesuses & 8 \\
\hline marchandes des modes / & \\
toiles/mercières & 4 \\
\hline modistes & 3 \\
\hline travailleures à la campagne/ maraîchères & 3 \\
\hline quincaillières & 2 \\
\hline revendeuse des légumes et fruits & 2 \\
\hline revendeuses des comestibles & 2 \\
\hline sage-femmes & 2 \\
\hline travailleuses en cuir et peaux & 2 \\
\hline aubergistes & 1 \\
\hline fabricantes des bas & 1 \\
\hline fabricantes des dentelles & 1 \\
\hline femmes au foyer & 1 \\
\hline marchandes & 1 \\
\hline ménusier & 1 \\
\hline quincallière et loue des chambres & 1 \\
\hline revendeuses de farines, graines et ris & 1 \\
\hline serveuses & 1 \\
\hline & \\
\hline métier non connu & 109 \\
\hline tot & 146 \\
\hline
\end{tabular}

Le moindre poids des couches moyennes et supérieures ne signifie pas que leurs membres ne recouraient pas à l'aliénation ; en réalité, vu les montants en jeu, ces groupes sociaux se tournaient plutôt vers le tribunal du Sénat de Piémont - la magistrature la plus importante du royaume -, comme nous avons pu le constater en consultant ses archives ${ }^{17}$.

Parmi les 144 cas de dots aliénées dont la valeur est connue, 57\% environ atteignaient 500 livres et environ 24\% s'élevaient à 1500 livres ou plus. Sans vouloir

17 Le Sénat du Piémont était la plus haute magistrature civile et criminelle de l'État; les juges étaient les sénateurs du royaume. AST, Senato di Piemonte, Licenze per alienare e permutare beni, ipoteche e ragioni dotali, Cat. XVIII. 
tirer trop vite des conclusions générales à partir de ces dots qui ont été constituées sur une longue période (dès les années 1730 et jusqu'à la fin du XVIII ${ }^{\mathrm{e}}$ siècle), on peut toutefois noter qu'une telle échelle de valeurs confirme les résultats obtenus par une autre étude : dans un échantillon de 735 dots catholiques constituées à Turin pendant le XVIIIe siècle, plus de $50 \%$ avaient une valeur comprise entre 1 et 499 livres $^{18}$. Les recours à l'aliénation dotale, on le voit, ne concernaient pas des couples particulièrement démunis. Ils touchaient au contraire de manière équilibrée l'ensemble de la population turinoise.

De la même manière, la liste des groupes professionnels concernés par les aliénations dotales reflète la structure socio-économique de la ville à l'époque. Selon le recensement de l'année 1802, environ $40 \%$ de la population participait à la production manufacturière, artisanale et au commerce ${ }^{19}$. Le secteur des services manuels - gagnedeniers, serviteurs et servantes, cochers, portefaix, palefreniers, porteurs, blanchisseuses et repasseuses, parmi tant d'autres - employait autour de $30 \%$ de la population. Environ $7 \%$ des habitants étaient occupés à des emplois d'écrivain, d'avocat, de notaire, de caissier, ou de fonctionnaire dans les bureaux de l'État. Les militaires constituaient quant à eux autour de $3 \%$ de la population, tandis que nobles, rentiers, religieux et religieuses en rassemblaient environ $7 \%$.

\section{La dot comme ressource}

Essayons maintenant d'analyser les raisons qui poussaient les couples à vendre et à utiliser leurs biens dotaux.

Plusieurs études, à la suite du fameux livre de Natalie Zemon Davis (1987), ont mis en avant le rôle fondamental du langage, de la présentation de soi et de son propre cas dans les procédures de la justice d'Ancien Régime. Il s'agissait d'un langage de communication avec les institutions qui, outre l'explication et la mise en relief de ce que les individus envisageaient, devait tenir compte de la politique des institutions, de ce que celles-ci voulaient bien entendre des individus. Bien évidemment donc, dans les

\footnotetext{
18 Parmi un échantillon de 735 dots catholiques enregistrées auprès des notaires turinois, 50,34\% ont une valeur allant jusqu'à 499 livres et 17,55\% entre 500 et 999 livres (Allegra, 1996, 177).

19 Le recensement de la ville de Turin de 1802, dont j'ai tiré ces données, a été saisi intégralement par une équipe d'étudiants coordonnée par Maria Carla Lamberti de l'Université de Turin (Département d'Histoire, section Histoire Économique), qui l'a généreusement mis à ma disposition.
} 
suppliques en question, l'accent était mis sur la nécessité de garantir sa survie et celle de sa famille, d'éviter de sombrer dans la mendicité, et de vivre dans des conditions dignes.

Pour sortir de l'impasse des suppliques et de leurs éventuelles stratégies rhétoriques, il est possible de considérer directement la nature des dettes que les couples devaient recouvrir avec l'argent dotal. En effet, dans l'acte notarié passé à la conclusion de la procédure juridique, le couple réglait les dettes éventuelles aux créanciers. Les actes étaient très détaillés à cet égard et on y retrouve des listes nominatives des créanciers ainsi que des récépissés des biens fournis. Dans notre échantillon, 98 actes sur 146 contiennent des quittances de paiement. Par ailleurs, ces paiements ne consommaient pas forcément tout le montant récupéré lors de l'aliénation, et si de l'argent était encore disponible, il était conservé. De même, s'il n'y avait pas de créanciers pressants et de dettes à recouvrir, les espèces étaient tout simplement encaissées par les conjoints (on retrouve cette situation dans 48 cas sur 146).

Les dettes impayées ainsi que les pressions des créanciers - et le risque d'être emprisonné - constituaient sans doute les hantises les plus répandues chez les ménages. Comme l'explique un certain nombre de recherches, les dettes créaient des liens entre le créancier et le débiteur et, surtout, un rapport de subordination que le créancier utilisait souvent à son propre profit pour instaurer une relation de dépendance et de contrôle de l'individu et de sa famille (Fontaine, 1994). Il était donc dans l'intérêt des couples de limiter le plus possible ce rapport de dépendance.

Mais comment et pourquoi les couples se couvraient-ils de dettes ? Qui étaient leurs créanciers ? Dans le tableau 3, j'ai répertorié les causes d'endettement les plus fréquentes à partir des listes de créanciers des dossiers d'aliénation, et établi leur poids dans l'ensemble des 98 actes avec quittance de paiement.

\begin{tabular}{|c|c|c|}
\hline & $\%$ & n. \\
\hline emprunts d'argent auprès des particuliers & 40,8 & 40 \\
\hline $\begin{array}{l}\text { achat d'un fond de négoce ou de commerce / achat } \\
\text { d'une boutique ou d'un magasin* }\end{array}$ & 38,8 & 38 \\
\hline loyers impayés & 37,8 & 37 \\
\hline
\end{tabular}




\begin{tabular}{|c|c|c|}
\hline rachat des biens auprès des prêteurs sur gage** & 21,4 & 21 \\
\hline achat des comestibles (pain, vin, viande) & 16,3 & 16 \\
\hline achats d'autres biens (chaussures, vêtements..) & 6,1 & 6 \\
\hline achat médicaments / frais pour le chirurgien & 5,1 & 5 \\
\hline nourrice & 2,0 & 2 \\
\hline apprentissage enfants & 2,0 & 2 \\
\hline \multicolumn{3}{|c|}{$\begin{array}{l}\text { * dont } 3 \text { achats exclusivement reservés au fond de commerce } \\
\text { de la femme }\end{array}$} \\
\hline
\end{tabular}

Les données montrent une double fonction de la dot dans l'économie familiale, celle-ci apparaissant comme une ressource « au quotidien » face au marché du travail urbain et face aux pratiques du crédit.

Tout d'abord, il apparaît que presque la majorité des couples (38\% environ) était endettée auprès d'un fournisseur, d'un marchand en gros ou d'un artisan particulier qui leur avait vendu la boutique ${ }^{20}$. La dot était donc employée pour avoir accès au marché du travail et s'y maintenir, pour constituer ou améliorer un fond de commerce, pour démarrer une activité. Il s'agit d'un modèle qui ne concernait que certains groupes sociaux, tels les artisans, les marchands et les revendeurs. Prenons l'exemple d'Emanuele et Lucia Maria Molo qui envisageaient d'ouvrir et de gérer un café ; avec l'argent tiré de la dot, ils achetèrent à divers particuliers plusieurs meubles (un comptoir et un placard pour la vaisselle, trois tables, douze chaises) des pièces en cuivre et en faïence pour préparer et servir le café (y compris 3 cafetières), ainsi que 25 libbre de café « du Levant » et 10 rubbi de sucre auprès du marchand en gros Francesco Sellaro ${ }^{21}$.

On signalera cependant que les sources ne sont pas toujours d'une extrême clarté. Si dans certains cas, les termes des conjoints laissent entendre que l'argent était destiné à nourrir une activité exercée en commun, dans beaucoup d'autres cela n'était pas aussi évident. Dans trois situations observées, la fourniture remboursée concernait explicitement une activité gérée par la femme seule : c'est ainsi par exemple que le

\footnotetext{
20 Comme j'ai pu le vérifier, certains des couples qui aliénaient les biens dotaux pour acheter un fond de négoce, s'engageaient explicitement devant la loi à garantir la dot sur le fond ou la boutique ainsi constituée.

$21 \quad$ AST, Notai di Torino, I vers., vol. 2754, ff. 476r-486r.
} 
couple Nagi paya 548 livres aux marchands en gros Silvestro et Brachetto pour acquitter des passementeries et d'autres biens destinés à l'activité de marchande mercière de Costanza, femme Nagi ${ }^{22}$.

Le tableau 3 montre ensuite que, dans les mêmes proportions ( $40 \%$ des couples environs), la dot jouait un rôle spécifique dans les circuits de crédit. En effet, ce capital servait pour recouvrir des emprunts d'argent contractés auprès de particuliers (notamment le chef de la boutique où le mari travaillait, ou plus fréquemment des notables), ou encore, dans plus de $21 \%$ des cas, pour racheter des vêtements, des linges et des meubles mis en gage auprès des «banchi feneratizi » gérés par les juifs dans le ghetto de la ville ou auprès des Monti di Pietà catholiques.

Ces dernières années, plusieurs études ont attiré l'attention sur la diffusion du petit crédit dans la vie quotidienne des sociétés de l'Ancien Régime et sur les circuits relationnels, de confiance et/ou de dépendance qui étaient à la base de celui-ci (Fontaine, 1994 ; Gambetta, 1997 ; Muldrew, 1998). Dans la gestion de l'économie familiale, ces emprunts étaient sans doute essentiels, mais s'ils restaient impayés trop longtemps, ils risquaient de rendre le couple insolvable - et donc de lui faire perdre tout crédit - et, par là même, de fragiliser ou de détruire ces circuits de confiance. L'aliénation de la dot, parce qu'elle permettait au couple d'obtenir assez vite des espèces et d'acquitter ses dettes, était donc une ressource centrale pour huiler les rouages de ce mécanisme et en assurer le bon fonctionnement.

La portée de cette remarque est renforcée par la prise en compte des autres emplois de la dot et des autres causes d'endettement. De manière significative, plus de $37 \%$ des couples utilisaient ce capital pour payer le loyer en retard ; tandis que $16,3 \%$ parvenaient ainsi à rembourser qui un boulanger, un commerçant ou un aubergiste, auprès desquels des achats de pain, de viande ou de vin avaient été faits à crédit. Enfin, $6 \%$ des couples réglaient des dettes pour des biens pris à crédit (notamment des chaussures ou des vêtements), et une autre 5\% celles contractées pour l'achat des médicaments ou après avoir eu recours aux services d'un chirurgien.

À la différence du premier modèle d'emploi, limité aux couches sociales de l'artisanat et du commerce, ce deuxième type d'utilisation des sommes récupérées lors de l'aliénation dotale concernait tous les niveaux de la société, y compris les fonctionnaires de l'État, les notaires et les avocats, pour lesquels la continuité de

22 AST, Notai di Torino, I vers., vol. 2778, ff. nn. 
l'emploi et la stabilité des revenus n'étaient pas plus assurées que pour les commerçants et les artisans, comme en témoigne le cas de l'avocat Francesco Giarij et de sa femme Elisabetta. Non seulement Francesco fut obligé de se déplacer pendant sa carrière d'une branche à l'autre de l'administration, mais ses revenus ne suffirent pas à garantir la survie de ses six enfants, tandis que son âge et sa faible santé ne lui permit pas d'augmenter son rythme de travail. De ce fait, pour les conjoints, le recours au crédit devint inévitable et, avec la dot récupérée en octobre 1767, ils s'empressèrent de payer le boulanger, de rendre de l'argent emprunté à Paolo Chiantore et de racheter des biens mis en gage ${ }^{23}$. Si, donc, la rareté de l'argent liquide intéressait tous les aspects de la vie quotidienne et obligeait les familles à vivre à crédit, de même, à tous les niveaux de l'échelle sociale (ou presque), l'emploi direct des biens dotaux était perçu comme une solution raisonnable pour faire face aux situations de difficulté économique.

\section{A quoi sert la dot? Quelques modèles}

À ce point de la réflexion, l'analyse des enjeux économiques de la dot ne serait pas complète si l'on ne prenait pas en compte la situation familiale des couples; dans ce but, je ferai ici appel à la notion de "cycle de vie »- largement employée par la démographie historique et l'histoire de la famille - afin d'esquisser quelques modèles.

Pour commencer, considérons le laps de temps écoulé entre la date (présumée) du mariage et celle de la demande d'aliénation ${ }^{24}$. Dans leurs suppliques, les conjoints décrivaient de façon détaillée l'histoire de la dot: qui l'avait constituée, quand et comment, qui l'avait payée (ou avait été censé la payer), où avait-elle été investie. Cela nous permet d'établir des corrélations entre le moment où le couple commençait sa vie commune et la date de la demande d'aliénation ${ }^{25}$. J'ai résumé les données dans le tableau 4.

\footnotetext{
23 AST, Notai di Torino, I vers., vol. 5166, ff. 124r-138r.

24 Dans les actes d'aliénation dotale, on ne connaît pas la date exacte du mariage, mais celle de la constitution dotale par acte notarié (si elle a eu lieu). Ici, j'ai considéré la date de la constitution de la dot comme assez proche de la célébration du mariage.

25 J'ai considéré uniquement les couples qui demandent l'aliénation dotale pour la première fois, c'est-à-dire 103 cas sur 146. Tous les autres déclarent avoir déjà dans le passé vendu une ou plusieurs parties des biens dotaux en suivant la même procédure.
} 


\begin{tabular}{|c|c|c|}
\hline \multicolumn{3}{|c|}{$\begin{array}{l}\text { Tab. 4. Délais entre la constitution de la dot } \\
\text { mariage) et la demande d'aliénation (103 } \\
\text { procédures) }\end{array}$} \\
\hline $\begin{array}{l}\text { date de la demande } \\
\text { d'aliénation }\end{array}$ & & \\
\hline $\begin{array}{l}3 \text { à } 15 \text { ans après le } \\
\text { mariage }\end{array}$ & 46 & 44,7 \\
\hline $\begin{array}{l}\text { plus de } 15 \text { ans après le } \\
\text { mariage }\end{array}$ & 22 & 21,4 \\
\hline $\begin{array}{l}1 \text { ou } 2 \text { ans après le } \\
\text { mariage }\end{array}$ & 15 & 14,6 \\
\hline $\begin{array}{l}\text { dot non costituée par } \\
\text { acte notarié }\end{array}$ & 14 & 13,6 \\
\hline inconnu & 6 & 5,8 \\
\hline tot & 103 & 100,0 \\
\hline
\end{tabular}

Parmi l'ensemble des couples décidés à vendre leurs biens dotaux, près d'un sur deux était marié depuis un délai allant de 3 à 15 ans environ (46 sur 103). Dans ce groupe, 25 couples avaient des enfants (dont plusieurs nourrissons); une femme était enceinte. Plus de 70\% étaient endettés ; il s'agissait sans doute des couples qui avaient recours à l'aliénation pour faire face aux déséquilibres qui se créaient entre ressources et taille du ménage au cours du cycle de vie de la famille, lorsque les enfants étaient encore trop jeunes pour contribuer activement au budget familial. La dot servait surtout pour payer les loyers, acheter de la nourriture et d'autres biens, mais aussi, dans le cas des artisans et des revendeurs, pour approvisionner l'atelier ou le commerce ou accroître son activité.

Ce dernier emploi, d'ailleurs, apparaît nettement prédominant parmi les 15 couples qui se situaient au tout début de leur vie conjugale (1 à 2 ans après le mariage) lors de la demande d'aliénation dotale. Ceux-ci utilisaient la dot presque uniquement comme forme d'investissement (9 sur 15). Pour ces derniers, la dot constituait donc surtout une ressource de longue durée et un véritable investissement, tandis que l'aliénation apparaissait comme un mécanisme spécifique d'autofinancement. Comme je l'ai dit, les actes notariés conservent toute la documentation nécessaire pour expliquer quand et à quel prix les produits, les matériaux et les biens avaient été achetés, et on 
peut penser que l'engagement du couple à solliciter l'aliénation des biens dotaux remplissait une fonction de garantie face aux fournisseurs et aux créanciers.

22 couples enfin vendirent leurs biens dotaux dans un autre moment-clé de leur cycle de vie, à savoir plus de 15 ans après le début de leur vie conjugale. Parmi eux, 11 se déclaraient âgés et voulaient utiliser la dot pour assurer leur survie dans la vieillesse, car ils se disaient désormais dans l'impossibilité de travailler. De plus, un tiers environ comptait encore des enfants à charge.

Ce qui ressort de l'analyse des actes est donc une utilisation diversifiée de la dot, qui s'articulait non seulement aux situations rencontrées par chacun des différents groupes socioprofessionnels mais aussi aux différentes phases du cycle de vie du couple, aux événements démographiques du ménage et aux conjonctures économiques.

En somme, la dot jouait le rôle essentiel d'une ressource qui pouvait être assez rapidement dépensée sur le marché. Le fait que le regard des chercheurs se soit porté en priorité sur la dot au sein des couches sociales élevées a contribué à produire et à diffuser une image spécifique de celle-ci en tant que patrimoine à thésauriser et à conserver dans le but de le transmettre aux générations suivantes. En revanche, les actes d'aliénations dotales montrent qu'à Turin dans la deuxième moitié du XVIIIe siècle, et pratiquement à tous les niveaux de l'échelle sociale, la dot formait une véritable ressource flexible et manipulable, et finalement un enjeu fondamental de l'économie domestique.

Cette transformation du patrimoine dotal en espèces signale d'ailleurs, sur la longue période, un processus d'affaiblissement plus général du système dotal en tant qu'institution. Il s'agit d'un phénomène qui devint très marqué à Turin au cours du $\mathrm{XVIII}^{\mathrm{e}}$ siècle, mais dont témoignent, sous des formes et des modalités différentes, d'autres recherches et d'autres terrains ${ }^{26}$.

\section{Les femmes mariées entre contraintes et libertés}

Revenons maintenant au point de départ de l'analyse, et à la question qui la parcourt plus ou moins explicitement. À qui dans le couple revenait la décision de recourir à l'aliénation ? Qui choisissait quoi ? Quel était le rôle de la femme mariée

\footnotetext{
$26 \quad$ C'est le cas par exemple des Pouilles (Delille, 1985) et de la Sicile (Fazio, 1992) entre le XVII ${ }^{e}$
} et le $\mathrm{XIX}^{\mathrm{e}}$ siècle. 
dans ces pratiques? Les aliénations dotales constituaient-elles un espace de revendication ou une autre preuve de son assujettissement à l'autorité maritale ?

Sans doute, la vente de la dot concernait-elle le couple : la femme ne pouvait pas la demander sans l'accord ou l'autorisation explicite du mari, qui en effet était présent dans la supplique au côté de sa femme ${ }^{27}$. D'ailleurs, si la vérification du consentement de la femme était nécessaire, elle était aussi reléguée à la dernière étape de la procédure, lorsque, après avoir reçu le décret formel du juge, les conjoints se trouvaient devant le notaire pour récupérer les espèces.

Les rares études qui ont abordé le sujet, en ont donné une lecture très négative et désavantageuse pour la femme. L'aliénation dotale serait surtout l'expression de l'autorité maritale sur le patrimoine de l'épouse ; le mari profiterait ainsi du capital dotal pour recouvrir ses dettes dues à une mauvaise gestion des affaires ou à des investissements malheureux (Bresnahan Menning, 1989 ; Fazio, 1996 ; Scardozzi, 1998).

Bien que, comme je l'ai montré, la dot ait été surtout destinée à autofinancer l'accès des conjoints au marché du travail et à payer les dettes de la survie au jour le jour, il est clair que des doutes demeurent à propos de la volonté réelle de la femme de vendre sa dot ; on peut légitimement se demander quels étaient la capacité de persuasion du mari et le poids de l'autorité maritale. Mais, d'autre part, il apparaît également nécessaire de comprendre si l'initiative de la femme jouait un rôle dans cette démarche. Ainsi, les actes d'aliénations nous interrogent sur le rôle actif ou passif de la femme dans cette procédure, et plus globalement, face aux dynamiques de la vie du couple et à la gestion du patrimoine.

Il ne faut pas pour autant sous-estimer la possibilité d'une stratégie synergique du couple ; le recours à l'aliénation pourrait donc être une démarche choisie en commun par les conjoints et faisant partie d'un projet plus large de survie ou d'amélioration des conditions de la vie familiale que le couple, dans un même mouvement, tentait par ce moyen de réaliser.

Il me semble en clair réducteur de considérer que le recours à l'aliénation dotale marque systématiquement la simple affirmation de l'autorité maritale sur le patrimoine de la femme. Il est susceptible de s'inscrire dans un ensemble de stratégies mises en place par le couple lui-même. Et rien ne permet d'affirmer qu'il y a forcément une

27 Sauf dans certains cas spécifiques sur lesquels je reviendrai plus loin. 
dimension conflictuelle entre les conjoints lors d'une aliénation dotale. Les indices qui témoignent de la variété des stratégies engagées sont d'ailleurs nombreux.

On aborde ici le délicat problème des opportunités et des possibilités d'action des individus, et en particulier face à la loi et aux institutions d'Ancien Régime ; il s'agit d'un problème historiographique complexe qui a été abordé par des recherches récentes ${ }^{28}$ et qui sera développé dans la dernière partie de cet article.

\section{Qui paie la dot? Institutions et individus}

Ces dernières années, plusieurs études ont soulevé la question cruciale de la capacité d'agir des individus et des familles. Ces travaux ont insisté sur leur capacité à dialoguer avec les institutions, à les interroger et à les utiliser selon un éventail de modes de relations qui dépasse l'opposition simple entre la pure conflictualité et la collaboration. Comme l'écrit Renata Ago, « it became increasingly clear that the relations between precepts and behavior could no longer only be conceived in terms of imposition, execution and deviance. Such a net alternative between adaptation and revolt was far too brutal and various sources showed that the choices available to the crowd were absolutely not so limited »(Ago, 2002, 12).

Les sociétés d'Ancien Régime se caractérisaient par l'existence de systèmes normatifs souvent contradictoires qui étaient le résultat d'une superposition et d'un mélange entre différents droits, coutumes et chartes statutaires. C'est là que selon certains historiens, les espaces d'action et de choix des individus résidaient. Dans ces interstices qui existaient entre les normes ou qui étaient créés par les normes ellesmêmes, diverses pratiques coexistaient ${ }^{29}$. Les aliénations des biens dotaux s'inscrivent parfaitement dans les cas d'incohérence des systèmes normatifs et témoignent de la capacité des individus à profiter de ces espaces pour prendre des initiatives et agir en ayant connaissance d'un certain nombre d'opportunités; et finalement, par le biais de ces mêmes institutions, de viser des objectifs qui n'étaient pas forcément ceux que celles-ci envisageaient explicitement.

\footnotetext{
28 Sur le rapport entre femmes, patrimoine et droits de propriété dans le contexte italien d'Ancien Régime, voir les recherches de Ago (1996; 1998), Arru (1998a), Bellavitis (2008), Chabot et Calvi (1998), Fazio (1992; 1999), Feci (2004) et Groppi (1996).

$29 \quad$ Voir les réflexions de Levi (1989) et Martinat (2009).
} 
Avant d'arriver à employer et/ou à réinvestir ses biens, le couple engagé dans une aliénation dotale, devait récupérer la somme concernée, c'est-à-dire avoir concrètement entre les mains le montant en argent liquide. Or, plusieurs indices suggèrent que cela était le véritable but de l'aliénation dotale : à savoir tout bonnement récupérer l'argent comptant. Dans ce contexte, il ne s'agissait pas d'une question anodine et le problème est justement de savoir à qui le couple devait s'adresser pour percevoir la somme.

Considérons les individus appelés devant le juge du tribunal de la Giudicatura civile de Turin afin de payer la dot aux conjoints qui avaient obtenu un décret d'aliénation (tableau 5).

Tab 5. Individus appelés en justice pour payer la dot lors de la
procédure d'aliénation (146 cas)

Les membres de la parenté - et notamment les parents du côté de l'épouse - y jouaient un rôle important; de manière très significative, 16 couples réclamaient le paiement de la dot au frère de l'épouse, 5 à son père, 2 à la mère veuve, 2 à l'oncle paternel et 1 respectivement à la soeur et à l'oncle maternel. Or, si la présence des membres de la parenté n'est pas forcement surprenante puisqu'il s'agissait d'une dot, il est essentiel de souligner que pour un bon nombre des couples, la récupération des biens 
dotaux coïncidait avec le paiement de la dot elle-même ; en d'autres termes, la demande d'aliénation était un moyen (ou, mieux, un expédient ?) pour récupérer concrètement la dot. Cela est tout à fait vraisemblable, car le paiement de la dot était souvent promis mais jamais effectué, et bien que la dot fût établie avec un acte public devant le notaire, dans la plus grande partie des cas, cela n'impliquait pas son paiement immédiat. Au contraire, parfois il existait un décalage temporel sensible entre la constitution officielle de la dot et le paiement concret de celle-ci, tandis que les écritures privées ou de crédit, à payer dans le futur et souvent avec des délais considérables, étaient très répandues. Force est donc de penser que dans nombre de situations, la procédure d'aliénation était en premier lieu un moyen pour récupérer la dot - ou du moins la partie qui n'avait pas encore été payée.

Bien entendu, la récupération des biens dotaux n'était pas l'objectif officiel que la jurisprudence piémontaise reconnaissait à l'aliénation, car on préférait mettre en avant la nécessité de subvenir aux besoins de la famille. Pourtant, la lecture des dossiers ne laisse pas de doutes : une partie importante des couples avait recours à l'aliénation, principalement pour réclamer et percevoir matériellement en espèces la dot déjà promise et jamais reçue. Cela n'empêchait pas que la dot fût réellement nécessaire aux urgences familiales et qu'elle fût donc employée par le couple ; les deux motivations pouvaient tout à fait cohabiter. Voyons quelques cas dans le détail.

En 1771, Bernardo et Giacinta Caciandra adressèrent une supplique au roi afin de demander l'aliénation de la dot. Les frères de la femme étaient impliqués, car c'est à eux que revenait le paiement en tant qu'héritiers universels du père. Giacinta s'était mariée en décembre 1770, mais ses frères, Giuseppe Antonio et Gaetano Magnetti, ne lui avaient pas payé immédiatement la dot, d'une valeur de 400 livres. En juin 1771, le couple s'adressa au roi en expliquant que la dot était nécessaire et indispensable pour que Bernardo pût continuer son activité de fabricant de matelas : «Si celui-ci ne peut pas utiliser les 400 livres pour acheter des laines et des toiles, il sera obligé d'arrêter son activité et par conséquent, de mettre en péril la stabilité économique de la famille ». Face à cette explication, le roi et le juge de la Giudicatura délivrèrent un décret d'aliénation qui obligea les frères à se rendre au tribunal et à remettre l'argent au couple. Cette somme fut immédiatement utilisée pour payer la fourniture de laine déjà achetée au marchand en gros Pietro Giacomo Righini ${ }^{30 .}$ 
Parfois, la réclamation de la dot à la famille d'origine de l'épouse était beaucoup plus explicite et le couple ne se souciait pas de le faire comprendre, comme c'est le cas de Nicola et Margherita Martinetto. Dans la supplique, datée de juin 1780, Margherita déclara que la dot, de 150 livres, aurait du être payée par ses frères sur l'héritage paternel, à l'occasion de son mariage célébré en 1778. Or, depuis deux ans et demi, la dot n'était pas encore payée car les frères estimaient que le couple n'était pas suffisamment établi ${ }^{31}$. Et pourtant, c'était bien pour cette raison que les conjoints demandaient à percevoir l'argent dotal. En effet, Nicola était soldat et vivait à Novare, où se trouvait son régiment; Margherita en revanche, démunie et avec un nourrisson à charge, habitait Turin. Le couple demandait donc de récupérer et d'utiliser les biens dotaux afin que l'épouse pût rejoindre son mari à Novare et y établir un petit commerce de produits alimentaires. Or, si la situation particulièrement critique du couple (faible revenu du mari, un enfant à charge, habitation séparée des conjoints) justifiait sans doute la demande d'aliénation, il n'est pas possible, à la lecture, de ne pas remarquer le ton de réclamation de la supplique. La requête de Margherita répondait non seulement à une nécessité contingente du cycle de vie familial, mais était directement et explicitement en relation avec le paiement du capital dotal. Par le biais de cette procédure, la femme envisageait de récupérer la dot qui, depuis le mariage, restait encore impayée. De plus, dans ce cas, à l'inverse de la situation précédente, les documents ne délivraient aucune information à propos du destin de l'argent récupéré ; on ne sait donc pas si cette somme fut dépensée, et si ce fut le cas, comment et dans quelle situation.

Le récit détaillé des étapes qui concernait la dotation, typique de ces suppliques, ainsi que la mise en relief du fait que le paiement n'avait pas eu lieu ou était soumis à la présentation de solides garanties, sont des informations apparemment très peu pertinentes quant à la demande d'aliénation proprement dite, dans laquelle le couple, dénonçant un état d'indigence, réclamait l'utilisation de l'argent dotal pour la famille. Pourtant, le décalage est apparent ; ces déclarations prenaient une signification spécifique lorsqu'on les lit à la lumière des dynamiques qui intéressaient le paiement

\footnotetext{
$31 \quad$ «..per essere predetta consupplicante istituita erede particolare dal di lei padre Giacomo Giordano nella somma di lire 150 che dovevano pagarsi al tempo del di lei matrimonio. E sebbene siano due anni e mezzo circa che contrassero essi il matrimonio, nondimeno non hanno ancora potuto conseguire detta somma, perché dovendo la medesima tener luogo di dote, intendono i fratelli della coesponente, eredi del loro padre, che venghi quella cautellata, locché non sono i ricorrenti in caso di fare ...» (c'est moi qui souligne) ; AST, Sez. Riun., Notai di Torino, I vers., vol. 2778, ff. 251r-254v.
} 
d'une dot. En faisant appel à des motivations familiales, le couple envisageait de fait d'obtenir la dot qu'il n'avait guère la possibilité d'obtenir autrement, face à des pères, oncles et frères récalcitrants à payer. Cela, bien entendu, sans pour autant exclure que le couple se trouvât réellement dans un état de nécessité. Il s'agissait donc de construire et de présenter tous les éléments et les preuves attestant les droits du couple sur les biens, afin d'en demander à coup sûr le paiement, sans risquer d'en retarder encore le versement ou d'ouvrir autres procédures et de faire face à de nouveaux frais.

« Sans aucun secours de la part de son mari »?

Les espaces d'actions des individus qui se dévoilent dans le choix d'utiliser l'aliénation comme moyen pour obtenir le paiement de la dot ne sont pas les seuls. En effet, il y avait des situations dans lesquelles la femme mariée avait le droit d'aliéner sans le consentement de son mari, lorsque celui-ci avait été jugé pour « vergentia ad inopiam», c'est-à-dire quand son train de vie avait été considéré à risque, et que l'administration du patrimoine (personnel et du couple), maladroite, représentait une véritable menace d'indigence. Cette situation était réglée par la jurisprudence : selon la Charte constitutionnelle, la femme avait le droit d'assurer la dot sur le patrimoine qu'il restait de son mari; de plus, c'était à elle qu'en revenait l'administration (Duboin, 1818-1869, Lib. VI, Tit. XIX).

Or, si la loi avait pour but de protéger le patrimoine féminin contre les gaspillages et une mauvaise gestion du mari, dans la pratique cela offrait à la femme une marge d'action plus importante, grâce à laquelle elle pouvait prendre des initiatives par rapport aux biens dotaux. Il était ainsi assez facile pour la femme de revendiquer le droit de gérer ses biens lorsque le mari était jugé « vergentem ad inopiam ».

Prenons le cas de Margherita Salar qui, en juin 1770, envoya une supplique au roi pour aliéner 2000 livres de sa dot. La supplique fut refusée et la femme invitée à se présenter de nouveau avec son mari. Ce qu'elle fit quelques semaines plus tard, lorsque le couple fit parvenir une nouvelle supplique. En effet, dans la première demande, Margherita se rendit seule chez l'avocat chargé de la rédaction. Elle se déclara femme de Giuseppe Pagano mais de fait, dans toute la supplique, elle utilisait la première personne. Margherita justifia sa demande par le fait qu'elle vivait séparée de son mari, à cause des maltraitances et de la mauvaise conduite de celui-ci, qui ne contribuait plus 
aux soins du ménage. Il avait été interné à l'Hôpital de la Charité, où il séjournait, tandis qu'elle se retrouvait seule avec un enfant à charge, devant assurer les dépenses de la famille. De plus, puisqu'elle vivait sans l'aide de son mari (« in nessun modo provvista dal detto di lei marito ", selon ses termes) elle avait cité son mari en justice (de nouveau auprès de la Giudicatura) pour réclamer la suspension de la gestion et de l'usufruit des biens dotaux par son mari. Dans sa supplique, Margherita faisait ainsi preuve d'une forte initiative personnelle dans sa revendication de libre utilisation de la dot; elle savait très bien que le manque d'aide de la part du mari lui permettait de prendre le contrôle sur les biens dotaux ${ }^{32}$.

À ces cas, on pourra assimiler celui des femmes abandonnées qui, de la même manière, ne pouvaient pas compter sur l'aide du mari ${ }^{33}$. En juillet 1770, par exemple, Maria Leijman, femme du notaire Gio' Filippo Fiando, s'adressa au roi pour aliéner 300 livres (la moitié de sa dot d'une valeur de 600 livres). Elle vivait séparée de son mari, car Gio' Filippo se trouvait dans une situation de grande pauvreté, « haillonneux » ( « cencioso »), et n'était pas capable de subvenir aux besoins de sa famille. C'était donc Maria, « abandonnée par son mari », selon son expression, qui contribuait aux frais du ménage : elle travaillait comme modiste, s'occupait des trois enfants et habitait chez sa mère ${ }^{34}$. On remarquera que cette femme déclarait vivre sans aucune aide de la part de son mari ; c'était là la condition qui permettait à Maria de réclamer la gestion et l'utilisation des biens dotaux. Dans ce cas également, la participation défaillante du mari aux charges de la vie conjugale - alourdie par son absence - constituait la raison qui suscitait des revendications sur le patrimoine dotal, même s'il n'existait pas de jugement officiel pour « vergentia ad inopiam ».

Ici, les opportunités d'action de la femme et ses revendications sur la gestion du patrimoine étaient liées à une situation de marginalité qui était définie par sa condition de femme mariée qui soutenait seule les charges du ménage et à laquelle était reconnu le droit de ne pas être assujettie à l'autorité maritale.

Mais dans plusieurs cas, des doutes persistent à propos de la situation réelle d'une femme soi-disant « abandonnée » ou d'un mari déclaré « vergentem ad inopiam ». En effet, des indices importants nous poussent à croire que ces conditions particulières

\footnotetext{
32 AST, Notai di Torino, I vers., vol. 2756, ff.nn.

33 Dans l'échantillon de l'analyse (146 procédures), j'ai repéré 10 cas de femmes seules ou abandonnées.

$34 \quad$ AST, Notai di Torino, I vers., vol. 2755, ff.nn.
} 
étaient parfois, mais pas toujours, des conditions fictives, mises en avant, une nouvelle fois, dans le but de demander et d'obtenir d'une façon légitime, le paiement d'une dot qui tardait à venir de la part de la famille de l'épouse ou encore une autorisation légitime d'emploi des biens dotaux.

Il suffit de feuilleter les archives notariales et des croiser les noms des couples pour prendre conscience de l'usage non anodin et encore moins naïf des institutions de la part des individus. On constate donc que, par exemple, la même Maria Leijman qui en 1770 s'était déclarée « abandonnée », envoya une deuxième demande d'aliénation en mai $1775^{35}$. Dans cette nouvelle supplique, qui visait à obtenir le reliquat de 300 livres de la dot, Maria Leijman était accompagnée par son mari qui vivait avec elle et ses trois enfants ; si le couple dénonçait encore la faiblesse de ses revenus, aucune allusion n'était faite à la condition de «femme abandonnée » et à l'absence du mari. Il est donc possible que, dans son cas comme dans d'autres qui lui ressemblent, la condition de «femme abandonnée» était largement fictive, et déclarée afin de pouvoir plus aisément - et avec le consentement de la loi - demander et employer les biens dotaux.

En définitive, l'analyse de ces suppliques et procès permet d'entrevoir un large éventail de dynamiques relationnelles et de stratégies individuelles et familiales. L'aliénation dotale avait lieu pour des raisons très différentes et pour répondre à des logiques et à des stratégies diversifiées qui concernaient, selon les cas, le mari, la femme ou les conjoints ensemble, et qui étaient destinées à assurer la survie de la famille, à soutenir une activité commerciale ou artisanale ou bien, tout simplement, à se faire payer en comptant la dot. Si donc, les protagonistes et les motivations pouvaient changer selon les contextes, force est de constater qu'une interprétation unique et uniformisante de toutes les procédures d'aliénation dotale n'est pas possible, ni crédible ; de même, il apparaît difficile de délimiter d'une façon univoque les espaces du choix du mari ou de la femme, ou encore du couple agissant d'un commun élan, vis-à-vis de la famille de l'épouse ou des créanciers de la dot.

\section{Conclusion}

L'analyse que nous venons de développer constitue une première tentative d'exploration d'un champ de recherches stimulant et prometteur, qui touche des enjeux 
historiographiques essentiels pour la compréhension des dynamiques familiales, économiques et relationnelles de la société d'Ancien Régime.

Premièrement, l'exploitation d'une source encore très peu connue par les historiens, les actes d'aliénation dotale, permet de saisir explicitement le rôle économique de la dot, souvent oublié au profit des qualités plus idéalisées et symboliques de ce capital. Lorsque l'on s'interroge sur les motivations qui sont en amont de la décision d'aliéner, on est obligé de constater leur complexité. Celles-ci peuvent être comprises en ayant connaissance du système de dotation au Piémont, des contraintes juridiques et des conditions de paiement des dots. En outre, la notion de « cycle de vie» se révèle ici un outil indispensable pour saisir la dot comme une véritable ressource familiale.

Par ailleurs, le recours à une action en justice et les ambiguïtés dont les procès d'aliénation dotales sont entourés permettent de réfléchir au rôle des conjoints dans cette démarche. Si l'historiographie traditionnelle a privilégié une lecture négative de l'aliénation, en tant que symptôme d'un processus de perte de contrôle de la femme mariée sur son patrimoine, une analyse plus contextualisée permet de dévoiler une réalité beaucoup plus nuancée dans laquelle stratégies familiales et individuelles se mélangent et s'entrecroisent. L'analyse des actes d'aliénation dotale montre comment les individus savent tirer profit des institutions - et surtout des espaces d'incohérence normative qu'elles laissent - pour viser à des buts qui ne sont pas forcement ceux établis par les institutions elles-même, ou pour le moins, qui ne sont pas établis de façon officielle. En conséquence, l'aliénation dotale apparaît comme un moyen pour accéder concrètement - et avec l'aval de la loi - à l'une des ressources parmi les plus importantes des familles (et souvent destinée à rester « virtuelle »).

En définitive, ces sources ambiguës montrent la difficulté à laquelle se heurtent les historiens et les historiennes lorsqu'ils s'interrogent sur la qualité des relations interpersonnelles au sein du groupe familial. Il s'agit de mécanismes qui restent difficiles à saisir mais qui, en restituant une image plus dynamique des couples de l'Ancien Régime, remettent en question certains stéréotypes et ouvrent de nouvelles pistes de recherche.

Beatrice Zucca Micheletto 


\title{
Résumé
}

L'objective de cet article est d'analyser le rôle économique de la dot dans les ménages turinois du XVIII ${ }^{\text {ème }}$ siècle. Propriété féminine par excellence, inaliénable, la dot était sous l'administration et le contrôle du mari pendant la vie conjugale. Cependant, par le biais d'une procédure juridique - connue comme aliénation dotale, les couples pouvaient aliéner ce bien et surtout le transformer en argent comptant. À l'aide d'un corpus significatif des procès d'aliénations, cette étude montre que le capital dotal était employé dans un large éventail des situations (paiement des dettes, démarrage ou élargissement d'une activité artisanale ou commerciale) tout au long du cycle de vie des couples. En outre, l'aliénation était une procédure qui visait tout simplement à accélérer le payement de la dot elle-même, très souvent promise et jamais payée, ainsi qu'en assurer son emploie libre et légal. Cela permet donc d'envisager les éventuels espaces d'initiative et de choix des couples (et notamment des épouses) vis-à-vis des leurs patrimoines.

\begin{abstract}
This article aims to investigate the economic role of dowry in households of $18^{\text {th }}$ century Turin. As we know, dowry was constituted by the bride and her family at the marriage. It belonged to the wife but was administrated by her husband during the marriage life. Furthermore, in theory, dowry could not be sold as it was inalienable. Despite of this, by means of a juridical procedure - known as alienazione dotale couples could alienate dowry in order to obtain cash. The analysis of a large sample of these civil courts allows to highlight how couples use or invest this capital to ensure their well-being and/or to settle into a professional position and according to their lifecycle. This study also shows that alienation was used by couples as a strategic way to accelerate payment of the dowry, often promised but never payed, and to freely and legally dispose of it. Finally, in this article we discuss possibilities and opportunities of choices and actions for couples (and moreover for wives) to manage their property.
\end{abstract}

\section{Bibliographie}

ABreu-FERreira, Darlene (2000), "Fishmonger and Shipowners : Women in Maritime Communities of Early Modern Portugal”, The Sixteenth Century Journal. The Journal of Early Modern Studies, vol. XXXI, 1, 7-23.

AGO, Renata dir. (1995), "Diritti di proprietà", Quaderni Storici, 88.

AgO, Renata (1996), "Oltre la dote. I beni femminili", 164-182, in Il lavoro delle donne, Angela Groppi (dir.), Roma-Bari, Laterza.

AGO, Renata (1998), "Universel/particulier: femmes et droits de propriété (Rome, XVII ${ }^{\mathrm{e}}$ siècle) ", Clio, 7, Femmes, dots et patrimoines, [En ligne], mis en ligne le 03 juin 2005. URL : http://clio.revues.org/index347.html. 
AGO, Renata (2002), "Introduction”, 6-29, in The value of the norm, Renata Ago (dir.), Rome, Biblink.

Ago, Renata, Borello, Benedetta dir. (2008), Famiglie. CirColazione di Beni, CIRCUITI DI AFFETTI IN ETÀ MODERNA, ROMA, VIELLA

ARRU, Angiolina dir. (1998a), "Gestione dei patrimoni e diritti delle donne”, Quaderni Storici, 98.

AllegrA, Luciano (1996), Identità in bilico. Il ghetto ebraico di Torino nel Settecento, Torino, Zamorani.

Beauvalet-Boutouyrie, Scarlett (2001), Etre veuve sous l'Ancien Régime, Paris, Belin.

Bellavitis, Anna (2001), Identité, mariage, mobilité sociale. Citoyennes et citoyens à Venise au XVI siècle, Rome-Paris, Ecole Française de Rome.

Bellavitis, Anna (2008), Famille, genre, transmission à Venise au XVI siècle, RomeParis, Ecole Française de Rome.

Bellomo, Manlio (1961), Ricerche sui rapporti patrimoniali tra coniugi, Milano, Giuffrè.

Bresnahan Menning, Carol (1989), "Loans and Favors, Kin and Clients: Cosimo de' Medici and the Monte di Pieta", The Journal of Modern History, 61 (3), 487-511.

CAlvi, Giulia, СНАвот, Isabelle dir. (1998), Le ricchezze delle donne. Diritti patrimoniali e poteri familiari in Italia (XIII-XIX secc.), Torino, Rosenberg \& Sellier.

Cavallo, Sandra, Warner, Lyndan dir. (1999), Widowhood in Medieval and Early Modern Europe, New York-London, Longman.

CERUTTI, Simona (2003), Giustizia sommaria, Milano, Feltrinelli.

CHABOt, Isabelle, FornASARI, Massimo dir. (1997), L'economia della carità. Le doti del Monte di Pietà di Bologna (secoli XVI-XX), Bologna, Il Mulino.

CHOJNACKI, Stanley (2000), Women and Men in Renaissance Venice : twelve essays on patrician society, London-Baltimore (Md.), John Hopkins University Press,

D'AMELIA, Marina (1990), "Economia familiare e sussidi dotali. La politica della confraternita dell'Annunziata a Roma (secoli XVII-XVIII)", 195-215, in La donna nell'economia. Atti della Ventunesima Settimana di Studi dell'Istituto Internazionale di Storia Economica "F. Datini”, S. Cavaciocchi (dir.), Firenze, Le Monnier.

DELILLE, Gérard (1982), “Un esempio di assistenza privata. I Monti di maritaggio nel Regno di Napoli (secoli XVI-XVIII)", 275-282, in Timore e carità. I poveri nell'Italia 
moderna. G. Politi, M. Rosa, F. Della Peruta (dir.), Cremona, Annali della Biblioteca Statale e Libreria civica di Cremona.

DeLILLE, Gérard (1985), Familles et propriété dans le Royaume de Naples (XVe-XIXe siècle), Rome-Paris, Ecole Française de Rome.

DiEFENDORF, Barbara (1982), "Widowhood and Remarriage in Sixteenth Century Paris", Journal of Family History, 4, 379-395.

DionisotTi, Carlo (1881), Storia della magistratura piemontese, 2 vol., Torino, Ed. Roux \& Favale.

DuboIn, Felice Amato (1818-1869), Raccolta per ordine di materia di leggi, editti, manifesti ecc...pubblicati...sotto il felicissimo dominio della Real Casa de Savoia, Torino, Marcio Tip.

ERICKSON, Amy Louise (2008), "Married women's occupations in eighteenth-century London", Continuity and Change, 23 (2), 267-307.

FAZIO, Ida (1992), "Valori economici e valori simbolici: il declino della dote nell'Italia dell'Ottocento", Quaderni Storici, 79, 291-315.

FAZIO, Ida (1996), "Percorsi coniugali nell'Italia moderna", 151-214, in Storia del matrimonio, M. De Giorgio, Ch. Klapisch-Zuber (dir.), Roma-Bari, Laterza.

FAZIO, Ida (1999), “Le ricchezze e le donne: verso una riproblematizzazione”, Quaderni Storici, 101, 530-550.

Fauve-Chamoux, Antoinette (2002), "Widows and their living arrangements in preindustrial France", Journal of Family History, 7 (1), 101-116.

FECI, Simona (2004), Pesci fuor d'acqua. Donne a Roma in età moderna: diritti e patrimoni, Roma, Viella.

FONTAINE, Laurence (1994), "Espace, usages et dynamiques de la dette dans les hautes vallées dauphinoises (XVII - -XVIII ${ }^{\mathrm{e}}$ siècles)", Annales HSS, 6, 1375-1391.

GAMBETTA, Diego dir. (1997), Le strategie della fiducia. Indagini sulla razionalità della cooperazione, Torino, Einaudi.

GoODY, Jack (1973), "Bridewealth and Dowry in Africa and Eurasia", 1-58, in Bridewealth and Dowry, J. Goody, S.J. Tambiah (dir.), Cambridge, Cambridge University Press.

GropPI, Angela (1994), I conservatori della virtù. Donne recluse nella Roma dei Papi, Roma-Bari, Laterza. 
GROPPI, Angela (1996), "Lavoro e proprietà delle donne in età moderna”, 199-163, in Il lavoro delle donne, A. Groppi (dir.), Roma-Bari, Laterza.

GropPI, Angela dir. (1998), CLIO. Histoire, femmes et sociétés, 7, Femmes, dots et patrimoines, [En ligne], mis en ligne le 03 juin 2005 . URL: http://clio.revues.org/index1324.html.

Howell, Martha (1998), The Marriage Exchange. Property, Social Place and Gender in Cities of the Low Countries, 1300-1550, Chicago-London, University of Chicago Press.

JURATIC, Sabine (1999), "Marchandes ou savantes? Les veuves des libraires parisiennes sous Louis XIV", 59-68, in C. Nativel (dir.), Femmes savantes, savoirs des femmes, Genève, Droz.

KLAPISCH-Zuber, Christiane (1983), "La mère cruelle. Maternité, veuvage et dot dans la Florence du XIV et XV ${ }^{\mathrm{e}}$ siècles", Annales ESC, 5, 1097-1109.

Klapisch-Zuber, Christiane (1990), "Le complexe de Griselda: dots et dons de mariage", 185-213, in La maison et le nom. Stratégies et rituels dans l'Italie de la Renaissance, Ch. Klapisch-Zuber, Paris, Ed. EHESS.

LANARO, Paola, VARANINI, Gian Maria (2009), "Funzioni economiche della dote nell'Italia centro-settentrionale (tardo medioevo/inizi età moderna)", 81-102, in La famiglia nell'economia europea, secc. XIII-XVIII, S. Cavaciocchi (dir.), Firenze, Firenze University Press.

LEVI, Giovanni (1976), "Terra e strutture familiari in una comunità piemontese del Settecento", Quaderni Storici, 33, 1095-1121.

LEVI, Giovanni (1989), Le pouvoir au village: histoire d'un exorciste dans le Piémont du XVIIe siècle, Paris, Gallimard (éd. italienne, 1985, L'eredità immateriale. Carriera di un esorcista nel Piemonte del Seicento, Torino, Einaudi)

MARTINAT, Monica (2009), “Individus et société, hier et aujourd'hui : quelques réflexions sur un couple problématique", 49-60, in Mobilité et transmission dans les sociétés de l'Europe moderne, A. Bellavitis, L. Croq, M. Martinat (dir.), Rennes, Presses Universitaires de Rennes.

MulDREw, Craig (1998), The Economy of Obligation. The culture of Credit and Social Relations in Early Modern England, Palgrave, New York.

OGILVIE, Sheila (2003), A bitter living : women, markets and social capital in Early Modern German, Oxford, Oxford University Press. 
Owen Hughes, Diane (1978), "From brideprice to dowry in Mediterranean Europe", Journal of Family History, 3 (3), 262-296.

Owen Hughes, Diane (1979), "Ideali domestici e comportamento sociale : testimonianze dalla Genova medievale", 147-183, in La famiglia nella storia. Comportamenti sociali e ideali domestici, C. E. Rosenberg (dir.), Torino, Einaudi.

PALAZZI, Maura (2002), "Economic autonomy and male authority : female merchants in modern Italy", Journal of Modern Italian Studies, 7(1), 17-36.

PELleGrin, Nicole, WinN, Colette dir. (2003), Veufs, veuves et veuvage dans la France d'Ancien Régime. Actes du Colloque du Poitiers (11-12 juin 1998), Paris, Champion.

PENE VIDARI, Gian Savino (1980-81), “Osservazioni sui rapporti patrimoniali fra coniugi nel Piemonte del sec. XVIII", Rivista di Storia del Diritto Italiano, 54, 19-60.

PENE VIDARI, Gian Savino (1983), "Dote, famiglia e patrimonio fra dottrina e pratica in Piemonte", 109-121, in La famiglia e la vita quotidiana in Europa dal '400 al '600, Actes du colloque International, Milan 1-4 décembre 1983, Roma, Ministero dei Beni Culturali.

SCARDOZZI, Mirella (1998), "Tra due codici: i contratti dotali nella toscana preunitaria", 95-120, in Le ricchezze delle donne. Diritti patrimoniali e poteri familiari in Italia (XIII-XIX secc.), G. Calvi, I. Chabot (dir.), Torino, Rosenberg \& Sellier.

Siegrist, Nora, SAmudio, Edda dir. (2006), Dote Matrimonial y Redes de Poder en el Antiguo Régimen en España y Hispanoamérica, Mérida, Venzuela, Telleres Graficos Universitarios.

VAn Den Heuvel, Danielle, VAn Nederveen MeERKerk, Elise (2008), "Introduction : Partners in business? Spousal cooperation in trades in early modern England and the Dutch Republic”, Continuity and Change, 23 (2), 209-216.

ZEMON DAVIS, Natalie (1987), Fiction in the Archives. Pardon tales and their tellers in sixteenth-century France, Stanford, Stanford University Press.

ZuCCA MiChELETTO, Beatrice (2008), "Lavoro femminile, figli ed economia domestica nella Torino di Antico Regime", Genesis. Rivista della Società Italiana delle Storiche, Flessibili/Precarie, VII, 1-2, 165-192. 